
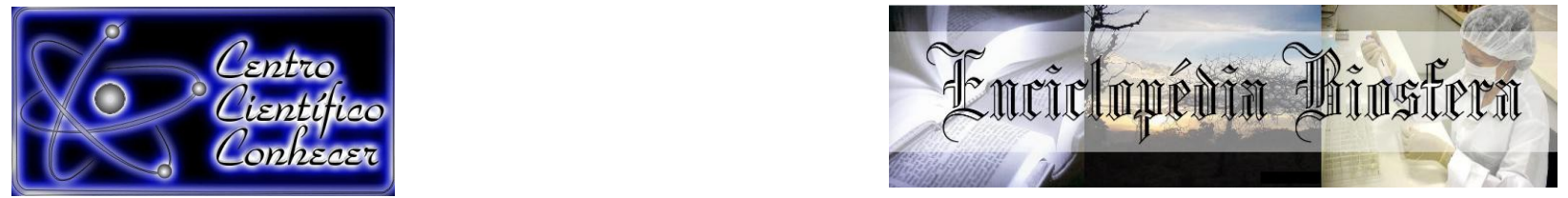

\title{
POTENCIAL DE IMAGENS MSI (SENTINEL-2) PARA CLASSIFICAÇÃO DO USO E COBERTURA DA TERRA
}

\author{
Franciel Eduardo Rex ${ }^{1}$, Pâmela Suélen Käfer ${ }^{2}$, Aline Bernarda Debastiani ${ }^{3}$, Verônica \\ Satomi Kazama ${ }^{3}$ \\ 1 - Mestrando do Programa de Pós-Graduação em Engenharia Florestal, \\ Universidade Federal do Paraná, Curitiba, PR - Brasil - francielrexx@gmail.com \\ 2 - Mestranda do Programa de Pós-Graduação em Sensoriamento Remoto e \\ Meteorologia, Universidade Federal do Rio Grande do Sul, RS - Brasil \\ 3 - Doutorandas do Programa de Pós-Graduação em Engenharia Florestal, \\ Universidade Federal do Paraná, Curitiba, PR - Brasil
}

Recebido em: 06/04/2018 - Aprovado em: 10/06/2018 - Publicado em: 20/06/2018 DOI: 10.18677/EnciBio_2018A67

\begin{abstract}
RESUMO
Há uma constante preocupação com a qualidade do mapeamento da cobertura terrestre, principalmente quanto ao refinamento e compatibilização da base de dados. Para tal finalidade, estão disponíveis gratuitamente imagens multiespectrais geradas a partir dos sistemas-sensores Landsat-8/OLI e Sentinel-2/MSI. Nesse sentido, o objetivo do presente estudo foi avaliar a qualidade da classificação dessas imagens de forma parcialmente não-supervisionada por meio do algoritmo cluster analisys, para retirar assim, a tendência do operador. Para tal, utilizou-se um recorte de uma área localizada no extremo Sul do estado do Rio Grande do Sul. As imagens adquiridas, foram obtidas nos dias 03 e 07 de janeiro de 2018. Foram utilizadas as bandas correspondes a faixa do visível, infravermelho próximo e médio, as imagens foram pré-processadas e classificadas de pelo algoritmo Cluster Analisys e posteriormente avaliadas pelo índice kappa (k). Em geral, a classificação da imagem Landsat 8 teve superestimativa de áreas nas classes ocupadas por campo e culturas anuais e subestimativa nas classes de agricultura, solo exposto, vegetação e água. A classificação da imagem Sentinel-2/MSI produziu resultados mais acurados $(\mathrm{k}=$ $0,98)$ em relação a do Landsat-8/OLI $(k=0,88)$. No entanto, ambas as classificações produziram resultados considerados como excelentes, ressaltando a qualidade que pode ser obtida a partir de imagens disponibilizadas gratuitamente.

PALAVRAS-CHAVE: Mapeamento, resolução espacial, resolução radiométrica.satélites.

\section{POTENTIAL OF IMAGES MSI (SENTINEL 2) FOR CLASSIFICATION OF EARTH USE AND COVERAGE}

\section{ABSTRACT}

There is a constant concern with the quality of the terrestrial coverage mapping, mainly regarding the refinement and compatibility of the database. For that purpose, multispectral images generated from the Landsat- 8/OLI and Sentinel-2/MSI sensor systems are available for free. In this sense, the objective of the present study was to evaluate the quality of the classification of these images in a partially unsupervised way by means of cluster analisys algorithms, to remove the tendency of the operator.
\end{abstract}


For that, a cut of an area located in the southern end of the state of Rio Grande do Sul was used. The images acquired were obtained on 03 and 07 of January 2018. The bands corresponded to the band of visible, near and medium infrared band were used, the images were preprocessed and classified by Cluster Analyzes algorithm and later evaluated by the index kappa $(\mathrm{k})$. In general, the Landsat 8 image classification had an overestimate of areas in the classes occupied by field and annual crops and underestimated in the classes of agriculture, exposed soil, vegetation and water. The classification of the Sentinel-2/MSI image produced more accurate results $(\mathrm{k}=0.98)$ in relation to that of Landsat-8/OLI $(\mathrm{k}=0.88)$. However, both classifications produced results considered as excellent, highlighting the quality that can be obtained from images made available for free.

KEYWORDS: Mapping, satellites, spatial resolution, radiometric resolution.

\section{INTRODUÇÃO}

O conhecimento sobre a condição do uso e cobertura da terra é uma informação essencial para uma variedade de necessidades sociais, que vão desde a gestão de recursos naturais, estudos ambientais, planejamento urbano, e desenvolvimento sustentável (ZELL et al., 2012; STERLING et al., 2013). A ciência sobre o uso da terra e a mudança da cobertura do solo, é uma das principais forças detectoras da mudança ambiental global, sendo fundamental para o debate sobre o desenvolvimento sustentável (HEGAZY et al., 2015).

Neste contexto, o sensoriamento remoto tem se destacado como uma ferramenta eficaz para o mapeamento da cobertura terrestre em larga escala (HANSEN et al., 2013) e tem sido amplamente utilizado na atualização dos mapas de uso e cobertura de terra bem como no mapeamento, tornando-se uma das aplicações mais importantes do sensoriamento remoto (LO; CHOI, 2004).

Por meio do uso de imagens de sensores remotos é possível realizar mapeamentos, avaliações e monitoramento da cobertura terrestre (BELWARD; SKØIEN, 2015; CHENG; HAN, 2016). Na literatura existe uma série de trabalhos que abordam o uso de sensoriamento remoto para o mapeamento do uso e cobertura da terra (por exemplo, DUSSEUX et al., 2014; STEFANSKI et al., 2014; ZHENG et al., 2015), nestas pesquisas, comumente é feita referência ao uso de imagens do satélite Landsat que representam registros valiosos e contínuos da superfície terrestre nas últimas décadas (USGS, 2018).

Dentre a série dos satélites Landsat, destaca-se o oitavo, lançado em 2013, é o mais avançado do que os demais. Tendo em vista que permite gerar imagens com resolução de $15 \mathrm{~m}$ coloridas por fusão digital e possui uma resolução radiométrica de 16 bits, diferente das demais séries anteriores que são limitados até $30 \mathrm{~m}$ e 12 bits de resolução espacial e radiométrica, respectivamente (USGS, 2015).Todo o arquivo Landsat se encontra disponível gratuitamente para o público científico, o que representa um poderoso banco de informações para identificar e monitorar mudanças nos ambientes físicos e artificiais (CHANDER et al., 2009; EL BASTAWESY, 2014).

Apesar disso, com o advento de novas tecnologias e melhorias no campo do sensoriamento remoto, torna-se necessário explorar novos meios de obter informações, neste aspecto, pode-se citar o novo satélite Sentinel-2 (S-2), um sensor multiespectral de resolução espacial média produzido pela Agência Espacial Européia (ESA). O S-2 possui um sensor com 13 bandas espectrais, de alta e média resolução espacial $(10,20$ e $60 \mathrm{~m})$ e resolução radiométrica de 12 bits (ESA, 2018), sendo capaz de alcançar alta resolução temporal (10 dias ou então 5 dias com dois 
satélites) que garante a continuidade dos dados necessários para o monitoramento global da terra (VAN DER MEER et al., 2014).

Métodos digitais de classificação de imagens de sensoriamento remoto têm adquirido uma importância crescente no reconhecimento automático de padrões da superfície terrestre (RICHARDS et al., 2005). Desta forma, tendo ciência que o Sentinel-2/MSI é um satélite recente, pesquisas a respeito do seu potencial ainda são escassas. Nesse sentido, o acesso liberado aos dados de sensores com características inovadoras impulsiona a realização e a investigação de novos estudos.

O objetivo do presente trabalho é avaliar o potencial de imagens do satélite Sentinel-2/MSI para classificação do uso e cobertura da terra, de forma não supervisionada através do algoritmo cluster disponível no software Quantum GIS (QGIS). A avaliação foi comparada com imagens do sensor Landsat-8/OLI , levando em consideração a resolução espacial e espectral.

\section{Caracterização da área de estudo}

\section{MATERIAL E MÉTODOS}

A área de estudo está localizada entre uma área de fronteira entre o Brasil e o Uruguai, extremo sul do Rio Grande do Sul e no nordeste do Uruguai (Figura 1).
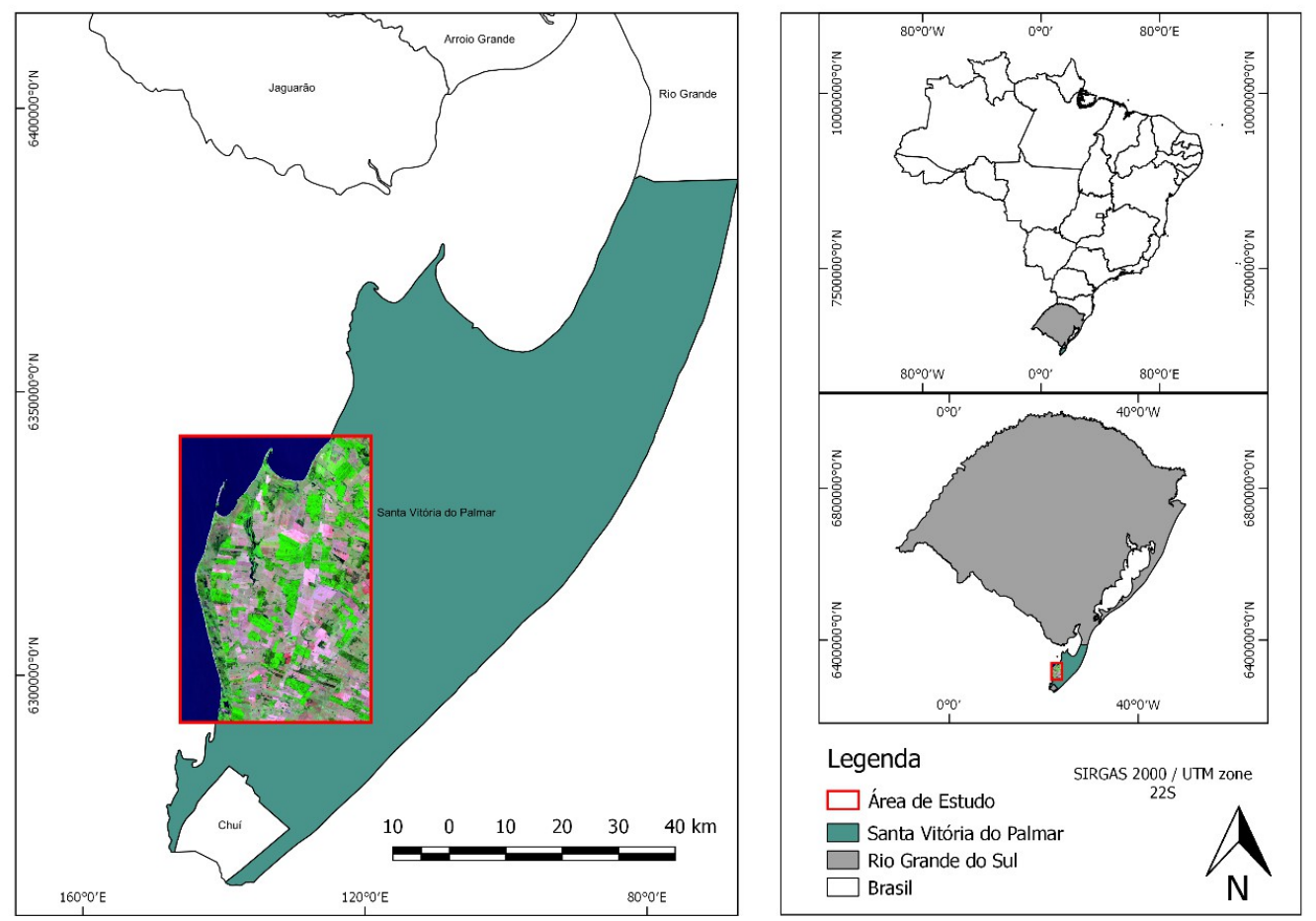

FIGURA 1: Localização da área de estudo no Brasil, no estado do Rio Grande do Sul, e na cidade de Santa Vitória do Palmar. Fonte: Os Autores (2018)

Dentre os municípios do Estado do Rio Grande do Sul, Santa Vitória do Palmar tem destaque por situar-se em uma região com um relevo de baixa rugosidade, com a presença de ventos intensos e constantes. Por tais motivos é considerada a área com potencial eólico mais promissora do Brasil. O município já possui os primeiros aerogeradores do Complexo Eólico Campos Neutrais instalados (CARDOSO; COLLISCHONN, 2015).

Conforme o último censo agropecuário pelo IBGE (2006), a utilização da terra do município conta com a presença de construções, benfeitorias ou caminhos $(2.665$ 
ha), lavouras (65.787 ha), matas e florestas (6.644 ha), pastagens naturais e antrópicas (24.255 ha), sistemas agroflorestais (1.567 ha), tanques, lagos, açudes e/ou área de águas públicas para exploração da aquicultura (662 ha), terras degradadas (erodidas, desertificadas, salinizadas, etc.) (1.868 ha) terras inaproveitáveis para agricultura ou pecuária (pântanos, areais, pedreiras, etc.) (10.266 ha).

\section{Sensores utilizados}

Para atingir o objetivo proposto foi efetuada a aquisição de imagens orbitais obtidas pelo sensor Multispectral Instrument (MSI), instalado a bordo do satélite Sentinel-2. Também foram adquiridas imagens do sensor OLI do satélite Landsat-8, as quais são comumente utilizadas para este tipo de estudo. O satélite Sentinel-2/MSI possui 13 faixas espectrais, semelhantes com as do Landsat-8/OLI, no entanto algumas bandas apresentam resoluções de $10 \mathrm{~m}$ - (ESA, 2018). As imagens foram adquiridas por meio do Serviço Geológico dos Estados Unidos (USGS) no endereço: http://earthexplorer.usgs.gov.

Optou-se por imagens mais recente e sem presença de nuvens. Para isso buscou-se imagens em período de seca na região, que ocorre entre dezembro e fevereiro. A imagem do sensor Landsat-8/OLI foi gerada no dia 07/01/2018, enquanto que a cena do Sentinel-2/MSI é datada em 03/01/2018. Para a avaliação foram escolhidas imagens de datas próximas para evitar a interferência de mudanças de uso da terra. Para o processamento das imagens foi utilizado o software livre QGIS 2.18. No quadro 1 são apresentas as descrições de cada sensor.

QUADRO 1: Descrições das bandas multiespectrais do sensor OLI/Landsat-8 e MSI/Sentinel-2.

\begin{tabular}{|c|c|c|}
\hline \multicolumn{2}{|l|}{ Sensor/Satélite } & \\
\hline $\begin{array}{c}\text { Landsat-8/OLI (comprimento de } \\
\text { onda central) }\end{array}$ & $\begin{array}{c}\text { MSI/Sentinel-2 } \\
\text { (comprimento de onda } \\
\text { central) }\end{array}$ & \\
\hline $\begin{array}{l}\text { Banda } 2 \text { - Azul } \\
\quad(483 \mathrm{~nm})\end{array}$ & $\begin{array}{l}\text { Banda } 2 \text { - Azul } \\
\quad(490 \mathrm{~nm})\end{array}$ & $\begin{array}{c}\text { Banda } 8 \text { - Infravermelho } \\
\text { próximo } \\
(842 \mathrm{~nm}) \\
\end{array}$ \\
\hline $\begin{array}{c}\text { Banda } 3 \text { - Verde } \\
(561 \mathrm{~nm})\end{array}$ & $\begin{array}{c}\text { Banda } 3 \text { - Visível Verde } \\
(560 \mathrm{~nm})\end{array}$ & $\begin{array}{c}\text { Banda 8a - Vermelho } \\
(865 \mathrm{~nm})\end{array}$ \\
\hline Banda 4 - Vermelho (654 nm) & $\begin{array}{l}\text { Banda } 4 \text { - Visível } \\
\text { Vermelho (665 nm) }\end{array}$ & $\begin{array}{c}\text { Banda 11- Infravermelho } \\
\text { Médio } \\
(1.610 \mathrm{~nm}) \\
\end{array}$ \\
\hline $\begin{array}{l}\text { Banda } 5 \text { - Infravermelho Próximo } \\
\qquad(864 \mathrm{~nm})\end{array}$ & $\begin{array}{c}\text { Banda } 5 \text { - Vermelho (705 } \\
\text { nm) }\end{array}$ & $\begin{array}{c}\text { Banda } 12 \text { Infravermelho } \\
\text { Médio } \\
(2.190 \mathrm{~nm})\end{array}$ \\
\hline $\begin{array}{c}\text { Banda } 6 \text { - Infravermelho Médio } \\
(1.609 \mathrm{~m})\end{array}$ & $\begin{array}{c}\text { Banda } 6 \text { - Vermelho (740 } \\
\text { nm) }\end{array}$ & 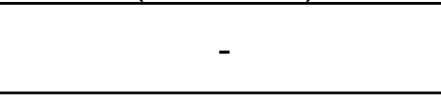 \\
\hline $\begin{array}{l}\text { Banda } 7 \text { - Infravermelho Médio } \\
(2.201 \mathrm{~nm})\end{array}$ & $\begin{array}{c}\text { Banda } 7 \text { - Vermelho (783 } \\
\text { nm) }\end{array}$ & - \\
\hline
\end{tabular}

Fonte: USGS (Science for a Changing World); e ESA (European Space Agency).

\section{Processamentos digitais}

Após a obtenção das imagens dos sensores Landsat-8/OLI e Sentinel-2/MSI, deu-se início a fase de pré-processamento. Nesta fase, as imagens foram submetidas à correção atmosférica e em seguida a reamostragem das bandas. Foram utilizadas as bandas 2, 3, 4, 5, 6 e 7 do Landsat-8/OLI, que possuem $30 \mathrm{~m}$ de resolução espacial, enquanto que do Sentinel-2/MSI foram utilizadas as Bandas 2, 3, 4, 8, com resolução de $10 \mathrm{~m}$, as Bandas 5, 6, 7, 08a, 11, 12, de $20 \mathrm{~m}$ de resolução. 
A correção atmosférica foi realizada para eliminar a interferência atmosférica pelo o método DOS1 (Dark Object Subtraction 1) por meio da ferramenta Semi Automatic Classification Plugin (SCP). A reamostragem das bandas foi realizada apenas para imagem Sentinel-2/MSI para compatibilizar todas as bandas em uma mesma resolução, ou seja, em um grid de $10 \mathrm{~m}$. Em seguida as cenas foram recortadas a partir de um retângulo de interesse, como apresentado na Figura 1.

Antes da aplicação do algoritmo classificador, foi realizada uma inspeção visual na área a fim de observar os principais alvos presentes na paisagem. Para isso, foram geradas composições coloridas do tipo (R)4 (G)3 (B)2 para ambos os satélites. Então foram identificadas seis classes de uso e cobertura da terra para a área de estudo, sendo elas: hidrografia, floresta, agricultura, pastagens, agricultura irrigada e solo exposto. O Quadro 2 ilustra a chave de interpretação dos alvos construída para auxiliar na definição das classes e posteriormente avaliação do resultado.

QUADRO 2: Chave de Interpretação para coleta das amostras de treinamento para a classificação das imagens.

\begin{tabular}{|c|c|c|c|}
\hline \multicolumn{4}{|c|}{ Chave de Interpretação dos Alvos } \\
\hline \multirow[b]{2}{*}{ Classes } & \multicolumn{2}{|c|}{ Satélite } & \multirow[b]{2}{*}{ Descrições } \\
\hline & $\begin{array}{c}\text { Landsat- } \\
8 / 0 \mathrm{LI}\end{array}$ & Sentinel-2 & \\
\hline Hidrografia & & & $\begin{array}{l}\text { Constituídos pelos cursos d'água de } \\
\text { rios, lagoas e os lagos artificiais } \\
\text { (reservatórios). }\end{array}$ \\
\hline Floresta & & & $\begin{array}{l}\text { Categoria de cobertura vegetal } \\
\text { natural, arbórea, representada por } \\
\text { vários tipos fisionômicos de florestas } \\
\text { e reflorestamento }\end{array}$ \\
\hline Agricultura & & & $\begin{array}{l}\text { Áreas cobertas predominantes por } \\
\text { algum tipo de vegetação do tipo } \\
\text { agrícola, ou, gramíneas, plantas } \\
\text { graminóides, etc. Não são cultivadas } \\
\text { por irrigação. }\end{array}$ \\
\hline Solo exposto & & & $\begin{array}{l}\text { Descaracterizado de cobertura } \\
\text { vegetal, solo com alto teor de argila, } \\
\text { apresentando alto teor de água. }\end{array}$ \\
\hline Pastagens & & a. & $\begin{array}{llll}\text { Vegetação } & \text { rasteira constituída } & \text { por } \\
\text { pastagens } & \text { (campos) naturais } & \text { e } \\
\text { antrópicas. } & & & \end{array}$ \\
\hline $\begin{array}{c}\text { Agricultura } \\
\text { irrigada }\end{array}$ & & & $\begin{array}{l}\text { Culturas agrícolas que são } \\
\text { submetidas à irrigação, muitas vezes } \\
\text { por inundação intermitente durante o } \\
\text { período de seca, destacadas pelo } \\
\text { vigor em relação as demais áreas. }\end{array}$ \\
\hline
\end{tabular}

Fonte: Os autores (2018).

A posteriori, as bandas dos respectivos sensores foram empilhadas e, desta forma, os conjuntos de dados foram submetidos a uma classificação parcialmente 
não$\begin{array}{lr}\text { não- } \\ \text { algoritmo cluster }\end{array} \quad K=\frac{P_{0}-P_{e}}{1-P_{e}}$ supervisionada. Para tal, foi utilizado o analisys, que está disponível no software do método não supervisionado está baseada em obter uma classificação padrão, sem tendenciosidade, ou seja, será aplicado o mesmo padrão de classificação para ambos os conjuntos de dados.

Para aprimorar a análise foram utilizadas informações auxiliares com o auxílio do aplicativo Google Earth, esse aplicativo foi utilizado como base para coletar amostras de campo para uma inspeção visual das classificações geradas. Nesse sentido, por se tratar de uma área relativamente pequena foram gerados 50 pontos aleatórios (Figura 2), pela ferramenta pontos aleatórios, que está disponível no software QGIS 2.18, para este procedimento, selecionou-se os limites compreendidos das imagens recortadas.

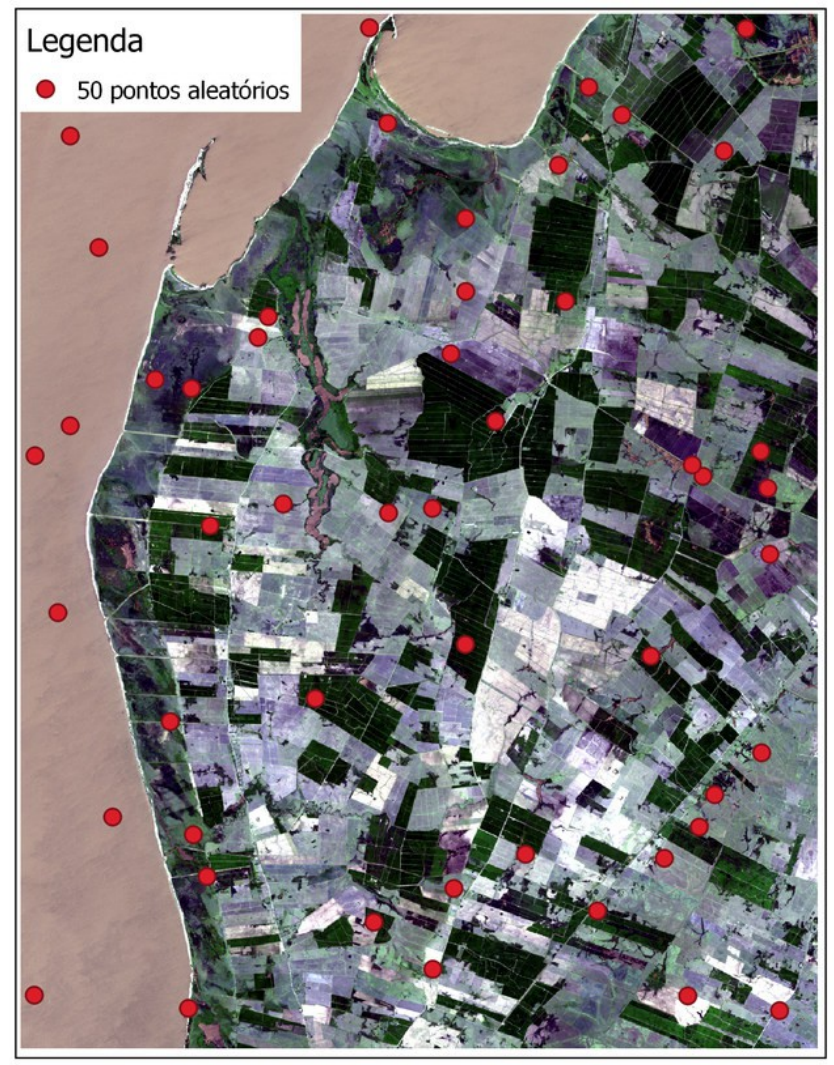

FIGURA 2: Distribuição dos 50 pontos aleatórios gerados para coleta das informações das classes, sob a imagem do sistema-sensor Sentinel-2/MSI. Fonte: Os Autores (2018).

Em seguida, as classificações foram avaliadas por uma matriz de confusão, exatidão global ( $G$ - Equação 1) e índice kappa ( $K$ - Equação 2$)$, os quais são utilizados para aferir a veracidade do resultado da classificação de uma imagem (MOREIRA, 2005).

$$
G=\frac{\sum_{i=1}^{M} n_{i i}}{N}=P_{0}
$$


(Equação

2)

Onde: nii = Número de pixels da diagonal principal da Matriz de Confusão de cada classe; $\mathrm{N}=$ Número de classes; $\mathrm{PO}=$ Concordância total ou Exatidão global; Pr: concordância ao acaso esperada; Pr: concordância esperada.

Conforme o índice Kappa, a classificação do mapa temático foi avaliada qualitativamente de acordo com a categorização proposta por Landis e Koch (1977). (Tabela 1).

TABELA 1: Qualidade da classificação associada aos valores da estatística Kappa

\begin{tabular}{lc}
\hline Valor de Kappa & Qualidade do mapa temático \\
\hline$<$ zero & Péssima \\
zero $-0,20$ & Ruim \\
$0,20-0,40$ & Razoável \\
$0,40-0,60$ & Boa \\
$0,60-0,80$ & Muito boa \\
$0,80-1,0$ & Excelente \\
\hline
\end{tabular}

Fonte: Adaptada de Landis e Koch (1977).

Após avaliação da qualidade das classificações foi realizada uma análise quantitativa em relação as áreas de cada classe em hectares (ha). Para tal aplicouse o teste de médias de Tukey, a 95\% de probabilidade para verificar se as classes obtidas pelas classificações dos satélites apresentam diferenças significativas.

\section{RESULTADOS E DISCUSSÕES}

$O$ algoritmo cluster analisys resultou em classificações visualmente semelhantes para as duas cenas, o resultado desta classificação pode ser observado na Figura 3.
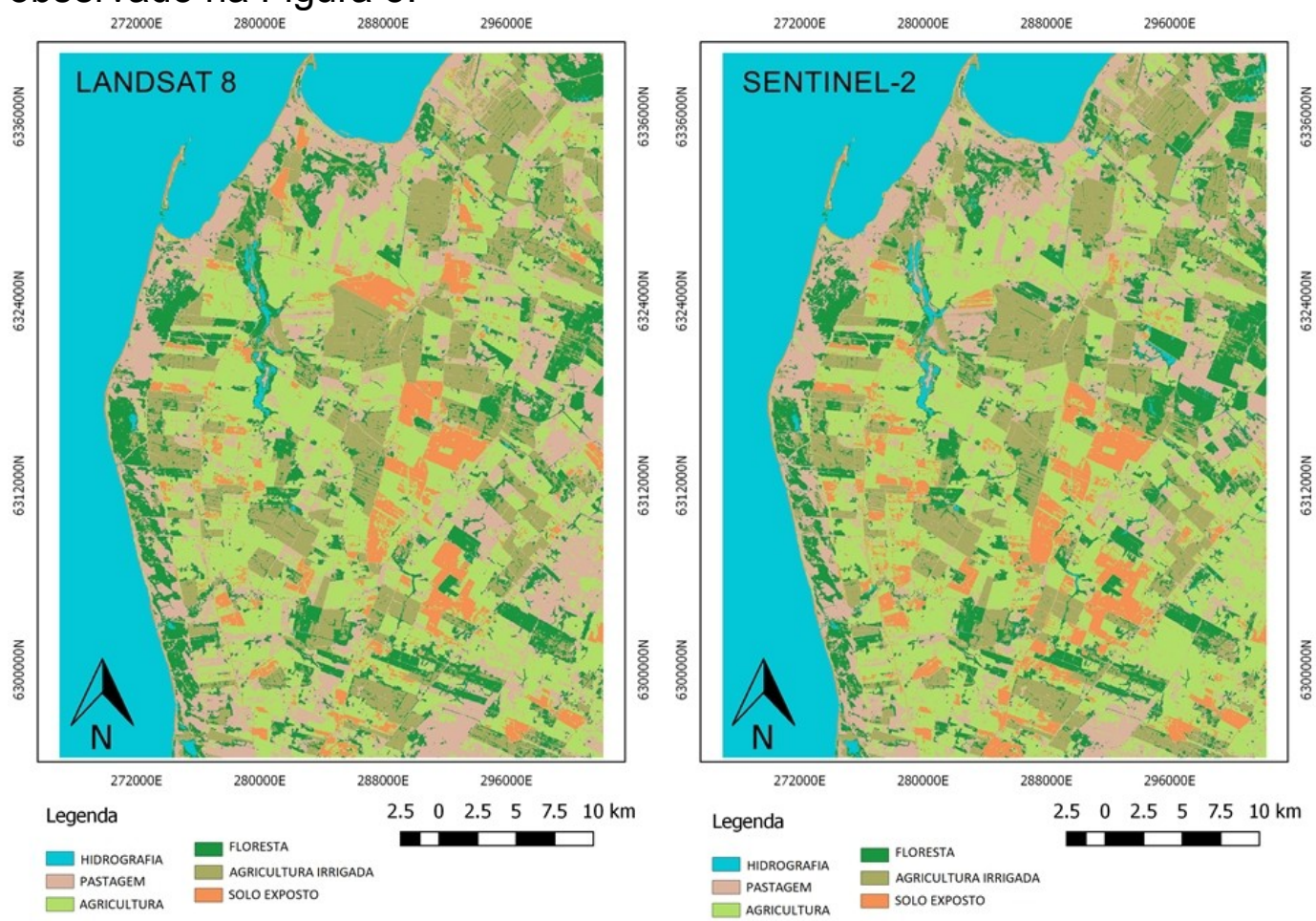
FIGURA 3: Classificação resultante para as imagens oriundas do Satélite Landsat-8/OLI e Sentinel-2/MSI. Fonte: Os Autores (2018).

No geral, os sensores conseguiram distinguir bem as classes propostas conseguindo representar bem a paisagem analisada. As classificações foram consideradas como excelentes ( $\mathrm{k}>0,81)$, conforme a classificação de Landis e Koch (1977), os valores encontrados de índice Kappa foram de 0,88\% para a imagem do satélite Landsat-8/OLI e de 0,98 para a imagem do satélite Sentinel-2/MSI.

Corroborando com tais resultados, um recente trabalho foi desenvolvido por IDE et al. (2017) na identificação de áreas irrigadas, para isso utilizaram de imagens do Sentinel-2/MSI e CBERS 4. Os autores encontraram valores de classificação considerados como sendo excelentes. Braz et al. (2017) também encontraram valores excelentes para o índice kappa na classificação de imagens Sentinel-2/MSI. Estes autores, no entanto utilizaram classificação supervisionada com o aporte do algoritmo Bhattacharya, onde é requerida a seleção de áreas de treinamento. Encontraram resultados superiores para a classificação da imagem do satélite Landsat-8/OLI em relação ao Sentinel-2/MSI. Uma possível suposição que pode explicar esse ocorrido, pode ter relação com processo de coleta de amostras de treinamento que pode ter influenciado nos resultados dos autores.

A resolução radiométrica inferior do satélite Sentinel-2/MSI em relação ao do satélite Landsat-8/OLI não influenciou no resultado da classificação, provavelmente o método de classificação abordado neste estudo, bem como a maior resolução espacial tenha conferido a superioridade no resultado. Em estudo de Sothe et al. (2017) foram encontrados valores superiores em imagens com melhor resolução radiométrica; os autores avaliaram imagens Rapidye e Landsat-8/OLI, e justificaram que a influência de sombras nas classificações das imagens RapidEye, conferiram a menor acurácia.

De acordo com Jensen (2011), uma alta resolução radiométrica geralmente aumenta a probabilidade de que os fenômenos sejam avaliados com mais exatidão pelo Sensoriamento Remoto, no entanto neste estudo a resolução radiométrica de 12 bits vinculada a resolução espacial de $10 \mathrm{~m}$ do sensor MSI do satélite Sentinel-2 revelou-se satisfatória para classificação do uso e cobertura da terra. A seguir, a Tabela 2 ilustra as matrizes de confusão das classificações geradas.

TABELA 2: Matriz de confusão em porcentagem gerada pela classificação das imagens oriundas dos satélites Sentinel-2/MSI e Landsat-8/OLI por meio dos 50 pontos amostrais

\begin{tabular}{|c|c|c|c|c|c|c|}
\hline \multicolumn{7}{|c|}{ SENTINEL-2 } \\
\hline Verdade de Campo & HD & $P G$ & AG & FL & $\mathrm{Al}$ & SE \\
\hline $\mathrm{HD}$ & 100 & 0 & 0 & 0 & 0 & 0 \\
\hline $\mathrm{CP}$ & 0 & 100 & 5,56 & 0 & 0 & 0 \\
\hline AG & 0 & 0 & 94,44 & 0 & 0 & 0 \\
\hline VG & 0 & 0 & 0 & 100 & 0 & 0 \\
\hline Al & 0 & 0 & 0 & 0 & 100 & 0 \\
\hline SE & 0 & 0 & 0 & 0 & 0 & 100 \\
\hline Total & 100 & 100 & 100 & 100 & 100 & 100 \\
\hline \multicolumn{7}{|c|}{ Landsat-8/OLI } \\
\hline Verdade de Campo & HD & PG & $A G$ & $\mathrm{FL}$ & $\mathrm{Al}$ & SE \\
\hline $\mathrm{HD}$ & 100 & 0 & 0 & 0 & 0 & 0 \\
\hline$P G$ & 0 & 66,66 & 16,66 & 0 & 0 & 0 \\
\hline AG & 0 & 33,33 & 83,33 & 33,33 & 0 & 0 \\
\hline $\mathrm{FL}$ & 0 & 0 & 0 & 66,66 & 0 & 0 \\
\hline $\mathrm{Al}$ & 0 & 0 & 0 & 0 & 100 & $\begin{array}{c}14, \\
3\end{array}$ \\
\hline
\end{tabular}




\begin{tabular}{lcccccc}
\hline SE & 0 & 0 & 0 & 0 & 0 & $\begin{array}{c}85, \\
7\end{array}$ \\
\hline Total & 100 & 100 & 100 & 100 & 100 & 100 \\
\hline
\end{tabular}

Fonte: Os autores (2018) em que: $\mathrm{HD}$ = hidrografia; $\mathrm{PG}$ = pastagem; $\mathrm{AG}$ = agricultura; $\mathrm{FL}$ = floresta; $\mathrm{Al}=$ agricultura irrigada; $\mathrm{SE}=$ solo exposto .

Em relação a matriz de confusão gerada a partir das classificações pode-se observar que o sensor OLI do satélite Landsat-8 apresentou as maiores confusões entre as classes. Esse resultado já era esperado, pois este sensor apresenta uma resolução espacial inferior $(30 \mathrm{~m})$, tendo um menor refinamento dos resultados em relação ao sensor $\mathrm{MSI}(10 \mathrm{~m})$.

Os maiores erros foram visualizados nas classes de agricultura e floresta. Para a classe de agricultura houve um erro de aproximadamente $17 \%$ com base nas amostras coletadas através do Google Earth, enquanto que para a classe de floresta o erro foi de aproximadamente $33 \%$, ou seja, das seis amostras de vegetação duas foram confundidas com a classe de agricultura.

Esta confusão pode ser justificada pelo fato de que as classes, floresta e agricultura são duas classes que se constituem de formações vegetativas e mesmo que se apresentem com componentes distintos, uma classe avança sobre a outra e não há uma divisão clara entre as mesmas. A transição de uma classe para outra causa a mistura de respostas espectrais no pixel, problema comentado no estudo de Mello et al. (2012). Cabe ressaltar que para a imagem Sentinel-2/MSI somente a classe agricultura apresentou confusão, sendo esta de apenas uma amostra, o que repercutiu em um erro de $5.56 \%$ com base nas amostras coletadas através do Google Earth.

Esses resultados revelam o potencial das imagens Sentinel-2/MSI para classificação do uso e cobertura da terra em relação as imagens Landsat-8/OLI, apesar de ambas terem sido classificadas como excelentes, é visível o melhor refinamento obtido com a imagem Sentinel-2/MSI. Nesse sentido, pode-se atrelar esse resultado com as características do sensor, como a melhor resolução espacial e o maior número de bandas.

Através do teste Tukey a 95\% de probabilidade foi observado que as classificações não apresentam diferenças significativas. A Tabela 3 apresenta os valores das áreas em hectares de cada classe bem como erro e desvio padrão entre as classes do uso e cobertura da terra.

TABELA 3: Composição de área, erro e desvio padrão em cada classe encontrada entre as imagens.

\begin{tabular}{lrrrr}
\hline \multirow{2}{*}{ Classes } & $\begin{array}{r}\text { Sentinel- } \\
\text { 2/MSI }\end{array}$ & Landsat-8/OLI & \multirow{2}{*}{ E.P (ha) } & Des. Pad \\
\cline { 2 - 3 } & Área (ha) & Área (ha) & & \\
\hline HD & 37725,10 & 37328,48 & 198,31 & 280,45 \\
\hline PG & 28885,45 & 32310,49 & 1712,52 & 2421,87 \\
\hline AG & 42773,31 & 40835,80 & 968,75 & 1370,03 \\
\hline FL & 20288,70 & 19725,70 & 281,50 & 398,10 \\
\hline Al & 22234,59 & 22270,25 & 17,83 & 25,22 \\
\hline SE & 9625,99 & 9115,19 & 255,40 & 361,19 \\
\hline
\end{tabular}

ENCICLOPÉDIA BIOSFERA, Centro Científico Conhecer - Goiânia, v.15 n.27; p. $227 \quad 2018$ 
Fonte: Os autores (2018), em que: E.P = erro padrão; Des.Pad = Desvio padrão; $\mathrm{HD}=$ hidrografia; $P G=$ pastagem; $A G=$ agricultura; $F L=$ floresta; $A l=$ agricultura irrigada; $S E=$ solo exposto.

É possível observar que de modo geral, os valores das classes se apresentam próximos entre si, sendo a classe campo com os maiores valores de desvio e erro padrão, para esta classe foram classificados aproximadamente 3400 ha a mais na imagem Landsat-8/OLI em relação a imagem Sentinel-2/MSI. Em seguida a classe agricultura apresenta os maiores valores de desvio e erro padrão, com aproximadamente 2000 ha a mais de áreas classificadas na imagem do Sentinel-2/MSI.

O menor erro é representado pela classe "Agricultura Irrigada", para esta, foram computados aproximadamente 35 ha a mais na imagem Landsat-8/OLI. Esses resultados fazem referência à matriz de confusão dos sensores, onde as maiores confusões estão atreladas aos maiores erros e desvios padrões da Tabela 3. Neste caso, pode-se citar que as classes de agricultura, floresta e pastagem, que foram as classes que apresentaram as maiores confusões (Tabela 2), o que corrobora com o resultado expresso anteriormente das áreas de cada classe.

Por fim, é ilustrada a Figura 4 que apresenta com melhor detalhe as classificações geradas para ambos os sensores. Nesta é possível visualizar com melhor rigor pequenas feições classificadas.

A)

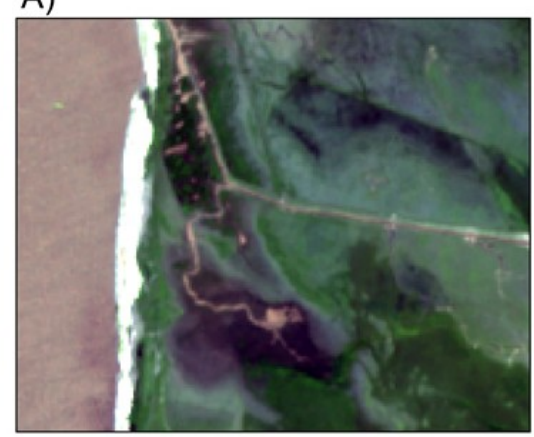

C)

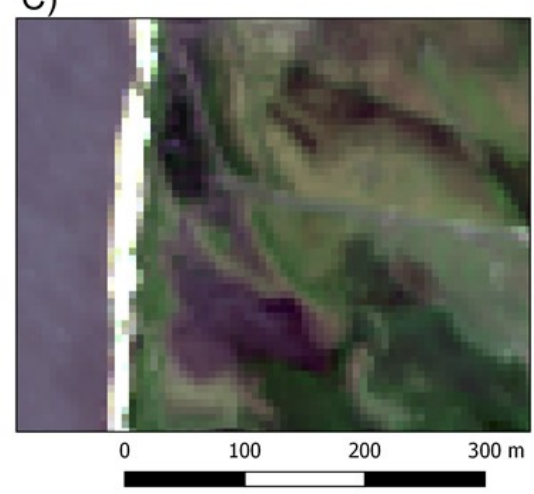

B)

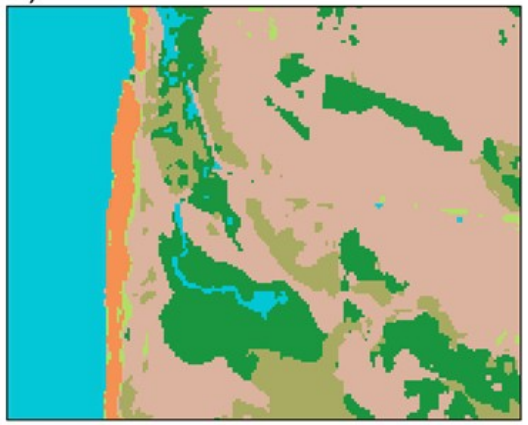

D)

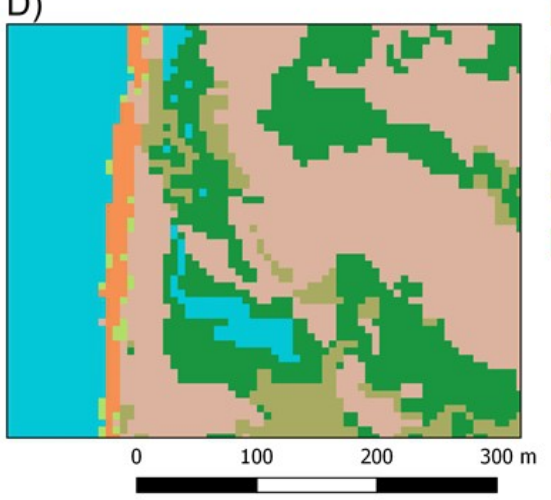

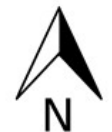

Legenda

HIDROGRAFIA

SOLO EXPOSTO

AGRICULTURA

PASTAGEM

AGRICULTURA

IRRIGADA

FLORESTA

FIGURA 4: (A) Destaque para a imagem Sentinel-2/MSI; (B) Imagem Sentinel-2/MSI classificada; e (C) Destaque para a imagem Landsat-8/OLI $\quad$ (D) Imagem Landsat-8/OLI classificada. Fonte: Os autores (2018).

\section{CONCLUSÕES}

As classificações não supervisionadas resultaram em maior exatidão para a imagem com melhor resolução espacial (10 m), satélite Sentinel-2/MSI $(k=0,98)$, 
quando comparada a classificação da imagem de média resolução espacial $(30 \mathrm{~m})$ Landsat-8/OLI $(k=0,88)$, porém ambas foram classificadas como sendo excelentes.

A classificação resultante da imagem do satélite Landsat-8/OLI teve superestimativa nas classes ocupadas por pastagens e agricultura irrigada e subestimativa nas classes de agricultura, solo exposto, floresta e hidrografia.

A resolução radiométrica inferior do sensor $\mathrm{MSI}$ do satélite Sentinel-2 (12 bits) em relação ao Landsat-8/OLI (16 bits) não comprometeu os resultados da classificação não supervisionada. Imagens do satélite Sentinel-2/MSI apresentam grande potencial para a classificação do uso e cobertura da terra, podendo este conjunto oferecer melhorias para o monitoramento da superfície terrestre.

\section{AGRADECIMENTOS}

Agradecimentos a Coordenação de Aperfeiçoamento de Pessoal de Nível Superior (CAPES) e o Conselho Nacional de Desenvolvimento Científico e Tecnológico (CNPq) pela concessão de bolsas.

\section{REFERÊNCIAS}

AGÊNCIA ESPACIAL EUROPEIA (ESA). Introduzindo o Sentinel-2. [2018]. Disponível em:

https://www.esa.int/Our_Activities/Observing_the_Earth/Copernicus/Sentinel2/Introducing_Sentinel-2>. Acesso em: 06 março. 2018.

BELWARD, A.S.; SKØIEN, J.O. Who launched what, when and why; trends in global land-cover observation capacity from civilian earth observation satellites. ISPRS Journal of Photogrammetry and Remote Sensing, v. 103, p. 115-128, 2015. Disponível em: https://doi.org/10.1016/j.isprsjprs.2014.03.009

BRAZ, A.M., BRAZ, A. M., BARROS, M. H. S., GARCIA, P. H. M. Validação das classificações das imagens dos satélites landsat 8 e sentinel-2 na bacia hidrográfica do córrego ribeirãozinho/ms. Revista Eletrônica AGB-TL, v. 1, n. 26, p. 47-58, 2017.

CARDOSO, A. M.; COLLISCHONN, E. Parques de produção de energia eólica e transformações na paisagem-estudo de caso em santa vitória do palmar/rs. Boletim Geográfico do Rio Grande do Sul, n. 25, p. 82-97, 2015.

CHANDER, G; MARKHAM, B. L.; HELDER, D. L. Summary of current radiometric calibration coefficients for Landsat MSS, TM, ETM+, and EO-1 ALI sensors. Remote sensing of environment, v. 113, n. 5, p. 893-903, 2009. Disponível em: https://doi.org/10.1016/j.rse.2009.01.007

CHENG, G.; HAN, J. A survey on object detection in optical remote sensing images. ISPRS Journal of Photogrammetry and Remote Sensing, 117, 11-28. 2016 Disponível em: https://doi.org/10.1016/j.isprsjprs.2016.03.014

DUSSEUX, P.; CORPETTI, T.; HUBERT-MOY, L.; CORGNE, S. Combined use of multi-temporal optical and radar satellite images for grassland monitoring. Remote Sensing, 6(7), 6163-6182. 2014. Disponível em: https://doi:10.3390/rs6076163 
EI BASTAWESY, M. Hydrological Scenarios of the Renaissance Dam in Ethiopia and Its Hydro-Environmental Impact on the Nile Downstream. Journal of Hydrologic Engineering, v. 20, n. 7, p. 04014083, 2014. Disponível em: http://dx.doi.org/10.1061/ (ASCE)HE.1943- 5584.0001112

HANSEN, M.C.; POTAPOV, P. V.; MOORE, R.; HANCHER, M.; TURUBANOVA, S.; Tyukavina, A.; ... \& Kommareddy, A. (2013). High-resolution global maps of 21stcentury forest cover change. Science, 342(6160), 850-853, 2013. Disponível em: DOI: $10.1126 /$ science. 1244693

HEGAZY, I. R.; KALOOP, M. R. Monitoring urban growth and land use change detection with GIS and remote sensing techniques in Daqahlia governorate Egypt. International Journal of Sustainable Built Environment, v. 4, n. 1, p. 117-124, 2015. Disponível em: https://doi.org/10.1016/j.ijsbe.2015.02.005

IBGE - Instituto Brasileiro de Geografia e Estatística (2006). Santa Vitória do Palmar: Pesquisas.Disponível em <https://cidades.ibge.gov.br/brasil/rs/santavitoria-do-palmar/pesquisa/24/27745>. Acessado em 14 marco 2018.

IDE, A. K.; ANDRADE, M. C. K. Impactos da perenização do Riacho Terra Nova na agricultura irrigada do município de Cabrobó/PE. Meio Ambiente $\mathbf{E}$ Sustentabilidade, v. 10, n. 6, 2017.

JENSEN, J. Sensoriamento Remoto do Ambiente - Uma Perspectiva em Recursos Terrestres. São Paulo:Parêntese, 2009.

LANDIS, J.; KOCH, G. G. The measurements of agreement for categorical data. Biometrics, v.33, n.3, p.159-179, 1977.

LO, C. P.; CHOI, J. A hybrid approach to urban land use/cover mapping using Landsat 7 Enhanced Thematic Mapper Plus (ETM+) images. International Journal of Remote Sensing, v. 25, n. 14, p. 2687-2700, 2004. https://doi.org/10.1080/01431160310001618428

MELLO, I. A. Y.; SALAS A. D. ALBUQUERQUE, L. C.; BUENO de L. F. Avaliação de técnicas de classificação digital de imagens Landsat em diferentes padrões de cobertura da terra em Rondônia. Revista Árvore, v. 36, n. 3, 2012. Disponível em: DOI: https://doi.org/10.1590/s0100-67622012000300016

MOREIRA, M. A. Fundamentos de Sensoriamento Remoto e Metodologias de Aplicação. 1.ed. São José dos Campos: INPE, 2001. 250 p.

PONZONI, F. J.; REZENDE, A. C. P. Influência da resolução espacial de imagens orbitais na identificação de elementos da paisagem em Altamira-PA. Revista Árvore, Viçosa, v. 26, n. 4, p.403-410, 2002. Disponível em: http://dx.doi.org/10.1590/S0100-67622002000400002.

RICHARDS, J. A. Analysis of Remotely Sensed Data: The Fomative Decades and the Future. IEEE Transactions on Geoscience and Remote Sensing, v. 43, n. 3, March 2005. Disponível em: DOI: 10.1109/TGRS.2004.837326 
SOTHE, C., LIESENBERG, V., de ALMEIDA, C. M., SCHIMALSKI, M. B. (2017). Abordagens para classificação do estádio sucessional da vegetação do parque nacional de são joaquim empregando imagens Landsat-8 e Rapideye. Boletim de Ciências Geodésicas, 23(3) 2017.Disponível em: http://dx.doi.org/10.1590/s198221702017000300026.

STEFANSKI, J., O. CHASKOVSKYY, B. W "Mapping and monitoring of land use changes in post-Soviet western Ukraine using remote sensing data." Applied Geography, Vol. 55, pp. 155-164, 2014 Disponível em: https://doi.org/10.1016/j.apgeog.2014.08.003

STERLING, S. M.; DUCHARNE, A.; POLCHER, J. The impact of global land-cover change on the terrestrial water cycle. Nature Climate Change, v. 3 , n. 4, p. 385 , 2013. Disponível em: DOI:10.1038/nclimate1690

USGS. United States Geological Survey. Free Data Proves Its Worth for Observing Earth. Disponível em: https://www.usgs.gov/news/free-data-proves-itsworth-observing-earth. 2018

USGS. United States Geological Survey. Landsat 8 OLI (Operational Land Imager) and TIRS (Thermal Infrared Sensor). Disponível em: https://lta.cr.usgs.gov/L8. 2015

VAN DER MEER, F. D.; VAN DER WERFF, H. M. A.; VAN RUITENBEEK, F. J. A. Potential of ESA's Sentinel-2 for geological applications. Remote sensing of environment, v. 148, p. 124-133, 2014. Disponível em: https://doi.org/10.1016/j.rse.2014.03.022

ZELL, E., HUFF, A. K., CARPENTER, A. T., FRIEDL, L. A. A user-driven approach to determining critical earth observation priorities for societal benefit. IEEE Journal of Selected Topics in Applied Earth Observations and Remote Sensing, 5(6), 1594-1602, 2012. Disponível em: DOI: 10.1109/JSTARS.2012.2199467

ZHENG, B., Myint, S.W.; Thenkabail, P.S.; Aggarwal, R.M. "A support vector machine to identify irrigated crop types using time-series Landsat NDVI data", International Journal of Applied Earth Observation and Geoinformation, Vol. 34, pp. 103-112, 2015. Disponível em: https://doi.org/10.1016/j.jag.2014.07.002 